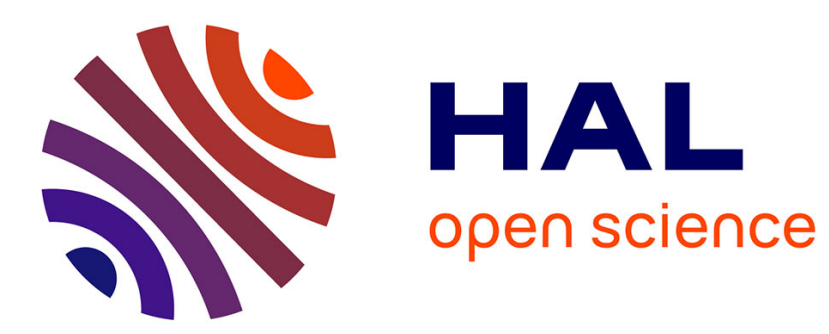

\title{
Segmentation of Kidneys Deformed by Nephroblastoma using Case-Based Reasoning
}

Florent Marie, Lisa Corbat, Thibault Delavelle, Yann Chaussy, Julien Henriet, Jean Lapayre

\section{- To cite this version:}

Florent Marie, Lisa Corbat, Thibault Delavelle, Yann Chaussy, Julien Henriet, et al.. Segmentation of Kidneys Deformed by Nephroblastoma using Case-Based Reasoning. International conference on Case-based reasoning, Jul 2018, Stockholm, Sweden. hal-02382619

\section{HAL Id: hal-02382619 https://hal.science/hal-02382619}

Submitted on 27 Nov 2019

HAL is a multi-disciplinary open access archive for the deposit and dissemination of scientific research documents, whether they are published or not. The documents may come from teaching and research institutions in France or abroad, or from public or private research centers.
L'archive ouverte pluridisciplinaire HAL, est destinée au dépôt et à la diffusion de documents scientifiques de niveau recherche, publiés ou non, émanant des établissements d'enseignement et de recherche français ou étrangers, des laboratoires publics ou privés. 
FEMTO-ST Institute

Univ. Bourgogne-Franche-Comté, CNRS

DISC, 16 route de Gray, 25030 Besançon, France

\{florent.marie,lisa.corbat, julien.henriet, jean-christophe.lapayre\}@univ-fcomte.fr CHRU Besanon

2 boulevard Fleming, 25000 Besanon, France

ychaussy@chu-besancon.fr 


\title{
Segmentation of Kidneys Deformed by Nephroblastoma using Case-Based Reasoning
}

\author{
Florent Marie $1 \quad$ Lisa Corbat1 Thibault Delavelle 1 \\ Yann Chaussy 2 Julien Henriet 1 \\ Jean-Christophe Lapayre 1
}

August 21, 2018

\begin{abstract}
Image segmentation is a hot topic in image processing research. Most of the time, segmentation is not fully automated, and a user is required to guide the process in order to obtain correct results. Yet, even with programs, it is a time consuming process. In a medical context, segmentation can provide a lot of information to surgeons, but since this task is manual, it is rarely executed because of time,. Artificial Intelligence (AI) is a powerful approach to create viable solutions for a fully automated treatments. In this paper, we defined a Case-Based Reasoning (CBR) which can enhance region-growing segmentation of kidneys deformed by nephroblastoma. The main problem with region-growing methods is a user needs to place the seeds in the image manually. Automated methods exist but are not efficient every time and often give an over-segmentation. That is why we have designed an adaptation phase which can modify the cordinates of seeds recovered during the retrievial phase. We confronted our CBR approach with manual region growing and Convolutional Neural Network (CNN) to segment kidneys and tumors of CT-scans. Our CBR system succeded in performing the best segmentation for the kidney. keywords: Case-Based Reasoning Convolution Neural Network segmentation cancer tumour healthcare imaging artificial intelligence
\end{abstract}

\section{Introduction}

Nephroblastoma, also called Wilms tumour, is one of the most frequently abdominal tumour observed in children. This type of tumour is situated in the kidney and its initial diagnosis is based on imaging. Radiologists and surgeons need 3-Dimensional (3D) representation of the tumour and the border organs in order to establish the diagnosis, plan the surgery, and also in order to gude the actions of surgeon during the surgery.

Segmentation is one of the key steps of the 3D construction. During this process, each pixel of every scans has to be affected to one, and only one, region. 
Radiologists and surgeons must guide and verify the segmentations manually. Our ambition is to introduce a process based on experts knowledge and experience in order to guide the segmentation process. This paper presents a CBR system which can enhance segmentations of kidneys deformed by nephroblastoma.

The originality of the present approach resides in the way our CBR system adapts the solutions of past situations. The principle of the region-growing segmentation consists of placing seeds on different points of the image that must be segmented, and then verifying if the pixels around these seeds have grey levels close enough to be integrated into the same region following thresholds. This algorithm makes each region grow until each pixel of the considered picture belongs to one, and only one, region. The main problem with the region-growing method is that a user needs to place the seeds in the image manually. Automated methods exist but are not efficient every time and often give an oversegmentation. Our idea consists in using a CBR system which places the seeds at the correct localisations, and then performing a region-growing segmentation. In addition, our system can modify the position of a seed during the adaptation phase in order to choose a pixel which matches the expected/average grey level of a kidney better. Many adaptation strategies can be found in the literature.

After this introduction, this paper presents the CBR system we have designed in order to compensate the unpredictability of tumoural kidney shapes: its case representation and all the phases of the system, putting the stress on the adaptation phase. The third part of this paper presents the performance of our method and these results are then discussed regarding the general purpose of the project.

\section{Related work}

There is a lot of research relative to segmentation enhanced by AI in literature. In [8] CBR is used with a watershed algorithm to segment pictures. Litjens et al. [18] wrote an survey for Deep learning in medical applications which is a mark of the popularity of Deep learning for segmentation. Other technics are also availables: genetic algorithm [10], knowledge stored in ontologies [26] or Random Markov Fields [15]. Deep learning (like Convolutional Neural Network $(\mathrm{CNN}))$ is one of the most efficient and promising tools, and it is widely used. CNN were used in order to perform segmentation of healthy kidneys [25, 3]. Nevertheless, They did not try to perform segmentations of kidney with tumours which introduces a difficulty. Actually, convolutional networks must be firstly trained to recognize the shapes of these organs. Since these two works focused on healthy organs, their shapes and areas are more or less the same from one subject to another. On the contrary, our work aims at performing segmentation of tumoral kidneys with unpredictable shapes, situation and very different form from a patient to another. Even if Deep learning seems to give the most accurate results in recent studies, this technique requires a lot of data in order to be 
trained. On the contrary, CBR takes advantage of knowledge and enriches itself following its experiments [16]. A large number of CBR systems designed for Health Science (CBR-HS) can also be found [6, 22, 20, 24, 2, 14, 13]. In particular, Perner designed a system for segmentation of brain images with a cut histogram method [23] and Frucci et al. adapted and improved this system with a watershed method [8]. We chose to implement a CBR system with a region growing method but, to place seeds at the optimum position, we need an adaptation phase.

Many adaptation strategies can be found in the literature. Adaptation using Adaptation Rules [21] consists of computing a solution for a target case applying a function which takes as parameters the target case, a source case that presents some similarities and its solution. Differential Adaptation [9] is based on the evaluation of the variations between the source and target cases: an approximate solution of the target case is computed by applying the variations between the two cases to the solution for the source case under consideration. Conservative Adaptation [17] is based on the Revision Theory which considers knowledge updates. This kind of adaptation consists of minimising the modifications to be applied to the knowledge and has been applied to the resolution of spatial and temporal problems [7] and also in oncology [5]. A. Cordier et al. [4] used Influence functions that link variations in problem descriptors to those in solution descriptors. In the CBR-HS EquiVox, an adaptation based on rules defined by experts experiences and Artificial Neural Networks (ANN) has been implemented and enhanced by a precision combination vector $[11,12]$. In the present work, the neighbourhood of the seeds are explored in order to match as much as possible to desired grey-levels. Consequently, our adaptation is a kind of Conservative Adaptation lead by rules.

\section{Materials and Methods}

This part of the paper presents the CBR system defined for image segmentation with regions-growing method, summarized in Figure 1. All the CBR phases are explained below. Our system comes from a CT-scan and looks for the closest stored image already segmented in the case base. It calculates a similarity value for each stored cases and extracts the source case with the highest similarity during a retrievial phase. Then, extracted parameters of segmentation are adapted to the current case through an adaptation phase. These adapted parameters are used to perform a new segmentation. Finally, the result is evaluated by an expert and stored in the case base as new source case if segmentation is relevant.

\subsection{Case model approach}

A case is composed of two parts : the Problem and the Solution. The Problem part describes the characteristics of the problem to be resolved, and the Solution part gives the way to solve it. The case model of the CBR is discribed in Figure 2 . 


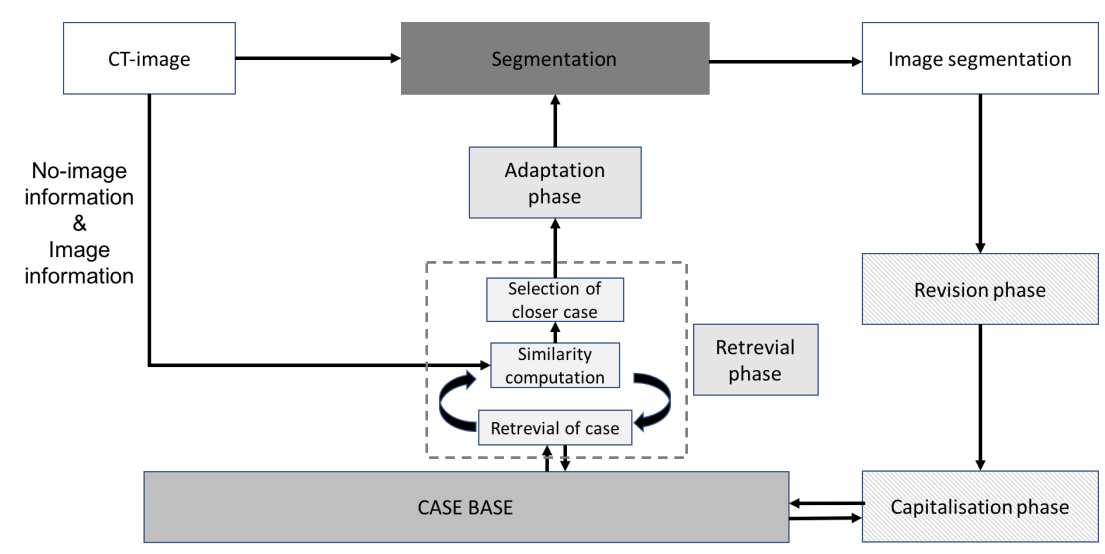

Manual phases

Figure 1: overview of our CBR system

In our study, the Problem part has to describe the CT-images, i.e. some descriptors that provide information about the structure or the statistics of the image. The Solution part should give the locations of seeds and the thresholds for the region-growing algorithm.

Like $[2,1]$, the Problem part of our cases is composed of :

- meta-data representing the information for the patient : sex, age, height and weight;

- statistical image information : mean, kurtosis, skewness and variance;

The Solution part of our cases contains the values of the thresholds (local and global) for each structure to segment, a list of positions for the seeds and a list of pretreatments (giving the order of the filters to apply and their parameters). We use three different pretreatments in order to enhance the contrast of the images : histogram equalization, median filter and unsharp mask. Each image has its own characteristics, thus it is necessary to add pre-processing to the Solution part, in order to fit each case.

\subsubsection{Retrieval phase}

This section describes how the retrieval phase is performed using a similarity calculation between the stored cases and the new problem.

As we explained in the last section, our case base is composed of two types of data: meta-data and image characteristics. We tested two variants of similarity formula between two images $x$ and $y$. First, we used Perner's formula as in [23], called $s_{1}(x, y)$ with a component $S M_{d}(x, y)$ for meta-data (a derivation of Tversky index) and another $S I(x, y)$ for image information. The second is a hybrid 


$$
\text { Case }=\left[\begin{array}{c}
\text { patient sex } \\
\text { patient age } \\
\text { patient height } \\
\text { patient weight } \\
\text { image mean } \\
\text { image kurtosis } \\
\text { image skewness } \\
\text { image variance }
\end{array}\right]+\left[\begin{array}{c}
\text { thresholds for each structure } \\
\text { list of pretreatments } \\
2 D \text { coordinates of kidney seeds }
\end{array}\right]
$$

\section{Description of problem part Description of solution part}

Figure 2: The case model of the CBR: problem part and solution part

formula called $s_{2}(x, y)$. It takes back the meta-data formula of Perner $S M_{d}(x, y)$ but uses MSSIM (Mean Structural SIMilarity) [27] criteria $(\operatorname{MSSIM}(x, y))$ for the image characteristics. The MSSIM criteria are an improvement of the SSIM (Structural Similarity) criteria that are commonly used in image compression. MSSIM uses an iterative windowing to increase the capacity of structural comparison on images (i.e. each window describes a ROI (Region Of Interest) which is compared independently). The following formulas show the construction of both similarity calculations between two images $x$ and $y\left(s_{1}(x, y)\right.$ and $\left.s_{2}(x, y)\right)$ :

$$
\begin{gathered}
s_{1}(x, y)=\frac{1}{2}\left(S M_{d}(x, y)+S I(x, y)\right) \\
s_{2}(x, y)=\frac{1}{2}\left(S M_{d}(x, y)+M S S I M(x, y)\right)
\end{gathered}
$$

The meta-data component is computed as follows:

$$
S M_{d}(x, y)=\frac{\left|A_{i}\right|}{\alpha\left|A_{i}\right|+\beta\left|D_{i}\right|+\gamma\left|E_{i}\right|}
$$

where $A_{i}$ is the number of common features between x and y, $D_{i}$ the features only in $x$ and $E_{i}$ the features only in $y . \alpha, \beta$ and $\gamma$ are weight factors such as $\alpha=\beta=1$ and $\gamma=0.5$.

The image component in $s_{1}(x, y)$ is:

$$
S I(x, y)=\frac{1}{K} \sum_{i=1}^{K} w_{i}\left|\frac{C_{i x}-C_{i m i n}}{C_{i m a x}-C_{i m i n}}-\frac{C_{i y}-C_{i m i n}}{C_{i m a x}-C_{i m i n}}\right|
$$

$C_{i x}$ and $C_{i y}$ are the $i$ th feature of both images $x$ and $y . C_{i m i n}$ and $C_{i m a x}$ are the minimum and the maximum of the $i$ th feature in the case base respectively. $w_{i}$ is a weight factor which allows to weight each image features. In our study, all weight factors are set to 1 .

The image component in $s_{2}(x, y)$ is: 


$$
\operatorname{MSSIM}(x, y)=\frac{1}{M} \sum_{i=1}^{M} \operatorname{SSIM}\left(x_{i}, y_{i}\right)
$$

where $M$ is the number of windows and SSIM is

$$
\operatorname{SSIM}(x, y)=\frac{\left(2 \mu_{x} \mu_{y}+C_{1}\right)+\left(2 \sigma_{x y}+C_{2}\right)}{\left(\mu_{x}^{2}+\mu_{y}^{2}+C_{1}\right)\left(\sigma_{x}^{2}+\sigma_{y}^{2}+C_{2}\right)}
$$

where $\mu$ is the mean, $\sigma$ is the standard deviation and $C_{1}=0.01 * L$ and $C_{2}=0.03 * L$ with $L=255$

\subsection{Adaptation phase}

The adaptation consists of positioning the seeds correctly. Since the tumour form and position are unpredictable, the position of the kidney is vulnerable to errors. Moreover, the kidney is a relatively small region, especially if the tumor crushes it. Consequently, the retrieved case is not always exactly the same as the new case we want to solve. So, the position of the seeds has to be adapted, especially for small regions.

We assumed that after the retrieval phase, the position of a seed is inside or close to its dedicated region. After applying our pre-treatments, we figured out that the grey-level intensity of an object to segment is almost always the same. Therefore, we can automatically infer the correct position of seeds, considering the grey-level intensity of the pixel. We defined a coherence interval for each small object to segment, corresponding to an interval of grey-level intensity in which a seed must be. We can define a procedure to verify if a seed belongs to its dedicated region by performing this test :

$$
\begin{gathered}
\forall \text { seed, isCorrectlyPlaced }(\text { seed })=\text { true } \\
\text { if } I(\text { seed }) \in C I_{\text {kidney }} / C I_{\text {kidney }}=[220,255]
\end{gathered}
$$

From this test, we defined an algorithm that can place the seed in a correct position on the image (i.e. in a pixel belonging to the kidney). Figure 3 illustrates the iterative extending of the neighbours from the initial postition of seed. The main idea is to evaluate the neighbours of the seed (8-connected), and check the coherence of these neighbours using the test presented above. If no candidate satisfies the test, we iteratively expand the scope of the neighbours until we find a coherent pixel. On Figure 3, each square represents a pixel of the image : in the first iteration we evaluate the direct neighbours of the white seed, in the second one we increase the scope to the pixels at a distance of two from the seed, and so on.

Giving a seed $S$ and a coherence interval $C I$, it returns the $2 \mathrm{D}$ coordinate representing the adapted position of the seed. For each evaluated neighbours, the test presented above is performed to check if the pixel is coherent, and at each iteration, the scope $(\alpha)$ is increased. 


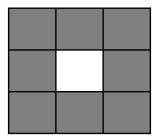

iteration 1

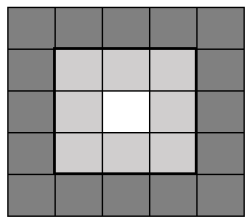

iteration 2

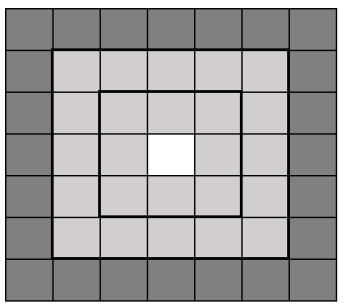

iteration 3

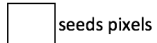

neighbours candidate pixels

already visited neighbours

Figure 3: Overview of the evaluated neighbours during the execution of the adaptation algorithm (for the position of the seeds) on 3 iterations

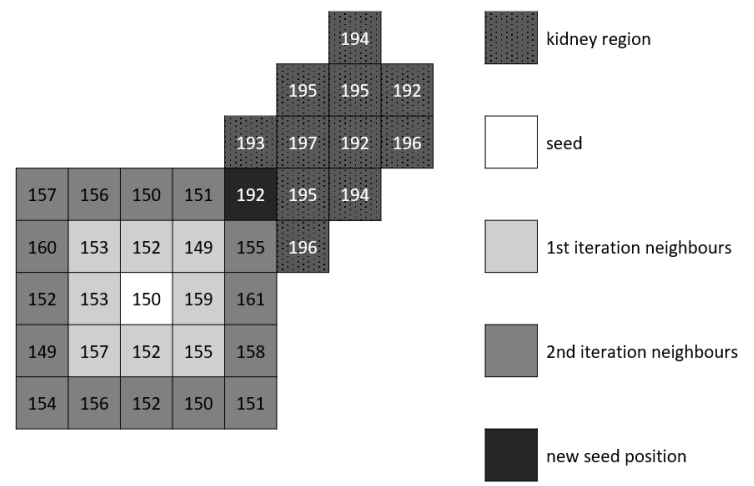

Figure 4: Execution example of the adaptation algorithm to find a new correct seed

Figure 4 shows an example of execution of the algorithm. From the initial seed (in white), we expand two times until a coherent candidate is found (the black pixel) that is part of the kidney region according to its grey level intensity $(>180)$.

\subsection{Revision and Capitalization phases}

As presented in Figure 1, all these phases are carried out by an expert. The expert evaluates the results of segmentation and decides to perform it again if necessary. The capitalisation phase is systematic, we enrich the case base after every segmentation obtained. Ideally, a satisfaction measure would determine if a resolved case could be added to the base. 


\section{Results}

We have tested the performance of our system on a set of 10 scans from one patient. We used a cross-validation method with a series of 10 tests. For each test, one image was used as target case, and the nine others were stored as source cases.

Then, we carried out tests in order to compare our approach with others for segmentation: region growing, watershed, threshold-based segmentation, and also FCN [19] which is a Convolutional Neural Network (deep-learning method).

\subsection{Coefficients for evaluation of results}

We used two scientific indicators in order to compare the results. The Dice and the IU. The Dice is commonly used by the experts of medical imaging, and the IU is an index commonly used by the Imaging community.

The DICE coefficient, also known as Sorensen coefficient, gives a similarity value (on $[0,1]$ ) between two sets $X$ and $Y$. In our case, $X$ represents the pixels of the ground truth (i.e. the desired segmentation) and $Y$ represents the pixels of the calculated segmentation given by our system. The above formula gives the details to calculate the value of the coefficient :

$$
D I C E=\frac{2 *|X \cap Y|}{|X|+|Y|}
$$

The IU (Intersection over Union, also called Jaccard index) is the mean of the $I U_{i}$ of all the regions, and the $I U_{i}$ of region $i$ is given by the equation below:

Where:

$$
I U_{i}=\frac{|X \cap Y|}{|X \cup Y|}=\frac{n_{i i}}{n_{i i}+n_{j i}+n_{i j}}
$$

- $n_{i i}$ is the number of pixels correctly placed in region $i$,

- $n_{j i}$ is the number of pixels that should have been in region $i$ but are not,

- $n_{i j}$ is the number of pixels that are in region $i$ but should not have been.

\subsection{Evaluation of similarity metrics}

Table 1 shows the differents similarity values according to the formulas mainly used in images processing with each source case. The last column shows the retrieved case for each target case and each similarity measure. The retrieved case is the case with the highest similarity value, excluding the target case (a case cannot retrieve itself). Results are equivalents for the both and, for each case, the retrivied case is the same. There are only two differences. On the one hand, Perner's formula seems more discriminative, with an higher difference beetwen the similarities from one image to another. But on the other hand, MSSIM seems more accurate because the similarity of two identical cases is equal to 1 . Consequently, the MSSIM similarity was chosen. 


\begin{tabular}{|c|c|c|c|}
\cline { 2 - 4 } \multicolumn{1}{c|}{} & Best $s_{1}$ & Best $s_{2}$ & Retrivied case \\
\hline Case 1 & 0.908 & 0.995 & 6 \\
\hline Case 2 & 0.945 & 0.995 & 7 \\
\hline Case 3 & 0.920 & 0.997 & 8 \\
\hline Case 4 & 0.929 & 0.997 & 9 \\
\hline Case 5 & 0.944 & 0.996 & 10 \\
\hline Case 6 & 0.908 & 0.995 & 1 \\
\hline Case 7 & 0.945 & 0.995 & 2 \\
\hline Case 8 & 0.920 & 0.997 & 3 \\
\hline Case 9 & 0.929 & 0.997 & 4 \\
\hline Case 10 & 0.944 & 0.996 & 5 \\
\hline
\end{tabular}

Table 1: Comparaison of two similarity measures (MSSIM and Attig and Perner's formulas) for each case during the cross validation

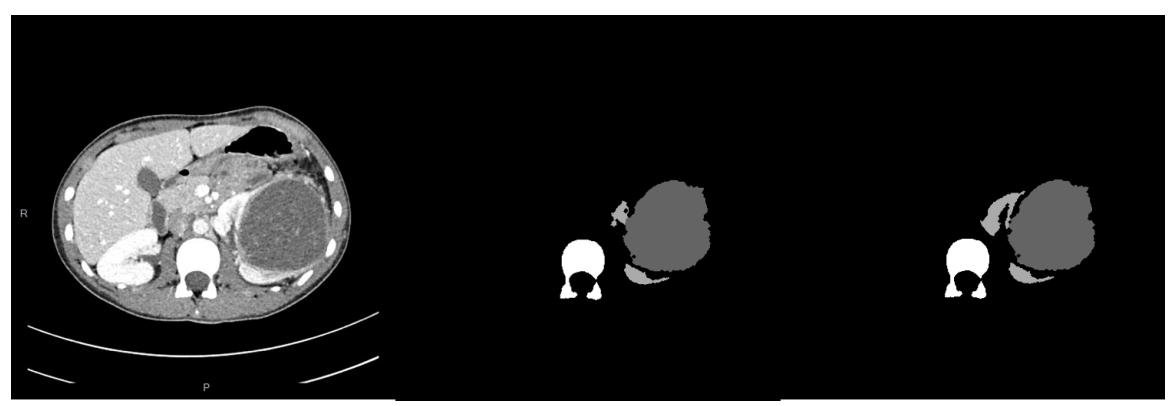

Figure 5: Comparison between an execution of our system with and without the adaptation phase (from left to right, CT-Scan, segmentation without adaptation and segmentation with adaptation)

\subsection{System adaptation}

In order to illustrate the effects of this adaptation algorithm, Figure 5 shows a comparison between an execution of our program with and without the adaptation phase. The kidney has a light grey label and the tumour has a dark grey label. From left to right we have a CT-image, segmentation without the adaptation phase, and finally segmentation with adaptation phase. For the segmentation without an adaptation process, the seed was placed outside the top part of the kidney. Consequently, the top part was not correctly segmented and the result has become irrelevant. Our adaptation phase helps to avoid this situation and improves the precision of segmentation.

Without adaptation, the seed was only placed at the correct position in 60 percent of our tested cases. Through the adaptation process, we improved the placement of seeds to 100 percent at the correct position.

Table 2 shows the results for kidney segmentation with DICE and IU. Using cross validation, the number corresponds to the tested scan as the target case, 


\begin{tabular}{|c|c|c|c|c|c|c|c|}
\cline { 2 - 8 } & Case & $\mathbf{1}$ & textbf0.9 2 & $\mathbf{0 . 9} \mathbf{3}$ & $\mathbf{4}$ & $\mathbf{5}$ & $\mathbf{0 . 9} 6$ \\
\hline \multirow{2}{*}{ CBR RG } & DICE & 0.998 & $\mathbf{0 . 9} 0.559$ & $\mathbf{0 . 9} 0.843$ & 0.894 & 0.997 & $\mathbf{0 . 9} 0.000$ \\
\cline { 2 - 8 } & IU & 0.995 & $\mathbf{0 . 9} 0.388$ & $\mathbf{0 . 9} 0.728$ & 0.809 & 0.995 & $\mathbf{0 . 9} 0.000$ \\
\hline \multirow{2}{*}{ CBR RG + adaptation } & DICE & 0.998 & $\mathbf{0 . 9} 0.999$ & $\mathbf{0 . 9} 0.993$ & 0.894 & 0.997 & $\mathbf{0 . 9} 0.998$ \\
\cline { 2 - 8 } & IU & 0.995 & $\mathbf{0 . 9} 0.999$ & $\mathbf{0 . 9} 0.987$ & 0.809 & 0.995 & $\mathbf{0 . 9} 0.997$ \\
\hline \multirow{2}{*}{ CBR RG } & Case & $\mathbf{7}$ & $\mathbf{8}$ & $\mathbf{0 . 9} \mathbf{9}$ & $\mathbf{1 0}$ & Mean & Median \\
\hline & DICE & 0.927 & 1.000 & $\mathbf{0 . 9} 0.936$ & 0.998 & 0.815 & 0.931 \\
\cline { 2 - 8 } & IU & 0.865 & 1.000 & $\mathbf{0 . 9} 0.879$ & 0.996 & 0.765 & 0.872 \\
\hline \multirow{2}{*}{ CBR RG + adaptation } & DICE & 0.927 & 1.000 & $\mathbf{0 . 9} 0.973$ & 0.998 & 0.978 & 0.997 \\
\cline { 2 - 8 } & IU & 0.865 & 1.000 & $\mathbf{0 . 9} 0.948$ & 0.996 & 0.959 & 0.995 \\
\hline
\end{tabular}

Table 2: DICE and IU for kidney segmentation with CBR-region growing

\begin{tabular}{|c|c|c|c|}
\cline { 2 - 4 } & Retrivied Case & Global error (\%) & Local error (\%) \\
\hline Case 1 & 6 & 0.0 & 0.0 \\
\hline Case 2 & 7 & 0.0 & 0.0 \\
\hline Case 3 & 8 & 0.0 & 0.0 \\
\hline Case 4 & 9 & 20.9 & 17.5 \\
\hline Case 5 & 10 & 3.6 & 0.0 \\
\hline Case 6 & 1 & 0.0 & 0.0 \\
\hline Case 7 & 2 & 0.0 & 0.0 \\
\hline Case 8 & 3 & 0.0 & 0.0 \\
\hline Case 9 & 4 & 29.7 & 14.5 \\
\hline Case 10 & 5 & 0.0 & 0.0 \\
\hline
\end{tabular}

Table 3: Mean errors for retrieved thresholds: local and global

with the nine others as source cases. We highlighted in grey cases presenting an improvement after the adaptation process. To check the efficiency of the adaptation process only, we compared CBR results with a ground truth, which is the result of a manual region growing. We highlighted in grey cases presenting an improvement after the adaptation process. Most of the time, the CBR process succeeded in recovering the correct coordinates to place a seed in the kidney. For these cases, adaptation did not improve the results because the CBR did not need it. However, some segmentations $(2,3,6$, and 9$)$ did not work well with just a CBR process and seeds were placed at the wrong coordinates (outside the kidney). Thanks to an adaptation step, we observed a clear improvement of results (until $99.8 \%$ for the Case 6 ).

Table 3 shows the error rates for each segmented case during the retrieval phase. Errors were computed with respect to thresholds determined during manual region-growing segmentation and are a mean between all seeds of the kidney (each seed has its own threshold and error rate). For most of them, we have a null error rate because the similarity between the problem case and the retrieved case is very high. So, thresholds are the same. But for some cases, we can observe an higher error rate (cases 4 and 9) from 14.5 to $29.7 \%$. It 
shows the main limitation of our CBR system without the adaptation phase for thresholds. This adaptation process represents an important perspective to improve its robustness. Case 7 has the lowest result in spite of an error rate of $0 \%$. It is because the used thresholds are closely related to the seed position. As the retrived position is not the same that seed position during manual region growing, it is logical that the scores are different. Furthermore, the manually determined parameters are not always the best possible. Thus, by coincidence, CBR solution can be better and has a score lower than 1 because of differences.

\subsection{Comparison with other approaches}

In order to compare our CBR system with other classical approaches, we carried out a segmentation with a manual level set with ImageJ software and a CNN. For the CNN, we implemented FCN-8s architecture from [19], trained with a training set of 10 slices (CT-Scans) corresponding to our case base and according to a cross-validation strategy to have the same conditions as CBR. Table 4 shows our results from these different methods. The CBR system version evaluated is the one with a Region-Growing (RG) and adaptation phase. As before, evaluation was done with a DICE and IU computation but, this time, we computed them from true ground truths carried out manually by paediatric surgeons.

As presented in Table 4, the best results were obtained with our CBR system. Indeed, we succeeded in having results very close to manual RG with a mean DICE of 0.83. A Level-Set technique shows good results for most of the cases, but it failed to segment Case 8 correctly. Globally, RG (manual or with CBR) performs more pertinent segmentations. $\mathrm{CNN}$ has the worst scores, with a mean DICE of only 0.59 . This is a logical result since CNNs are based on experiences and require a large database to yield interesting segmentations. CBR is another approach based on knowledge, which requires much less data in order to work well.

\section{Discussion}

Our results prove that our CBR system can enhance and guide segmentations of organs and structures deformed by a singularity (the tumour). Indeed, using this technique, the deformed tumoural kidneys of our scans were widely retrieved and better segmented than with all the other tested methods. This mainly comes from our adaptation phase ensuring that seeds are placed at the right place. Nevertheless, this adaptation is based on a coherence of pixel intensity and, unfortunately, different structures can have similar grey levels. Thus, it is possible that the adaptation selects the wrong pixel and places seeds in the spine or ribs, for example. Most of the time, kidneys are close enough to avoid this situation, but it could theoretically occur. In that respect, it would be interesting to enhance our adaptation phase to prevent this possibility. One way of doing that would be to drive the pixel research in the direction of kidney, rather than visiting all the neighbours around the initial seed. Another 


\begin{tabular}{|c|c|c|c|c|c|c|c|c|}
\cline { 2 - 9 } & \multicolumn{2}{|c|}{ CBR } & \multicolumn{2}{c|}{ RG } & \multicolumn{2}{c|}{ Level Set } & \multicolumn{2}{c|}{ FCN-8s } \\
\cline { 2 - 9 } & DICE & IU & DICE & IU & DICE & IU & DICE & IU \\
\hline Case 1 & 0.92 & 0.85 & 0.92 & 0.85 & 0.93 & 0.87 & 0.73 & 0.58 \\
\hline Case 2 & 0.89 & 0.80 & 0.88 & 0.79 & 0.88 & 0.79 & 0.78 & 0.64 \\
\hline Case 3 & 0.74 & 0.59 & 0.75 & 0.60 & 0.73 & 0.58 & 0.65 & 0.48 \\
\hline Case 4 & 0.74 & 0.59 & 0.79 & 0.65 & 0.75 & 0.60 & 0.52 & 0.35 \\
\hline Case 5 & 0.84 & 0.72 & 0.84 & 0.72 & 0.83 & 0.71 & 0.53 & 0.36 \\
\hline Case 6 & 0.95 & 0.90 & 0.95 & 0.90 & 0.90 & 0.83 & 0.47 & 0.31 \\
\hline Case 7 & 0.82 & 0.70 & 0.82 & 0.69 & 0.76 & 0.62 & 0.59 & 0.42 \\
\hline Case 8 & 0.76 & 0.62 & 0.76 & 0.62 & 0.18 & 0.10 & 0.60 & 0.42 \\
\hline Case 9 & 0.86 & 0.76 & 0.86 & 0.76 & 0.84 & 0.73 & 0.73 & 0.57 \\
\hline Case 10 & 0.76 & 0.61 & 0.77 & 0.63 & 0.73 & 0.58 & 0.35 & 0.21 \\
\hline Mean & $\mathbf{0 . 8 3}$ & $\mathbf{0 . 7 1}$ & $\mathbf{0 . 8 3}$ & $\mathbf{0 . 7 2}$ & $\mathbf{0 . 7 5}$ & $\mathbf{0 . 6 4}$ & $\mathbf{0 . 5 9}$ & $\mathbf{0 . 4 3}$ \\
\hline Median & $\mathbf{0 . 8 3}$ & $\mathbf{0 . 7 1}$ & $\mathbf{0 . 8 3}$ & $\mathbf{0 . 7 0}$ & $\mathbf{0 . 7 9}$ & $\mathbf{0 . 6 6}$ & $\mathbf{0 . 5 9}$ & $\mathbf{0 . 4 2}$ \\
\hline
\end{tabular}

Table 4: Comparison of our CBR system and some other approaches. DICE and IU were calculated for segmentation of deformed kidney

improvement would be the design of another adaptation phase for adjustment of the thresholds recovered from the source case.

Globally, RG produced better segmentations than the Level-Set (LS) technique although results are close for the most of cases. Yet, for some cases, LS parametrisation is easier and faster. This could permit designing a CBR system with both approaches: a case would be composed by the segmentation technique as well (RG or LS), following the best way determined during the manual step. In this way, a CBR system could retrieve the segmentation approach used to solve a case and not only the parameters of a particular technique. In our approach, CNNs have poor results because of the lack of data (only 10 images). The main advantage of CBR with respect to CNN is that CBR is simultaneously an experience approach and a knowledge approach, where CNN is just based on experience. This is the reason why CBR can perform interesting results even with a small data set. In addition, CNNs encounter many difficulties segmenting little structures, such as deformed kidneys, but could lead to results accurate enough with larger structures such as tumours. This helps to envisage a hybrid system, with a CBR system to segment kidneys and a CNN to segment tumours.

In addition, for surgeons, the segmentation of kidneys and tumours is not sufficient. Surgeons also need to visualise other small structures, such as blood vessels (artery, vein, etc.) in order to evaluate the difficulty of the surgery. Yet, it can be very difficult to segment them because of the lack of visibility. Due to their small size, placing a seed inside is not allowed. Deep learning could be a good option.

In regards with these results, inspired by [26], we want to go further and to integrate a real ontology which could guide the position of the seeds for all the other structures. 


\section{Conclusion}

We have designed a system based on CBR, which can guide segmentation of tumoural kidneys. We have also compared this approach to classical ones (commonly used in hospitals) and very recent ones (deep learning). These very promising results proved that we must delve further into this approach, and that we are on the correct path to automatic segmentation of all the organs in this part of the abdomen.

The difficulty of our problem resides in the fact that tumoural kidneys are deformed and moved by a totally unpredictable structure (the tumour). Nevertheless, our system can retrieve the positions of these organs.

Further work will consist of defining an ontology of this part of the body, including fuzzy information of the positions of the different structures. Moreover, an interesting future work would be the design of a hybrid segmentation system, with CBR and CNNs, to segment all of them.

\section{Acknowledgements}

The authors wish to thank Pr Frdric Auber, Dr Yann Chaussy, Dr Marion Lenoir-Auber of the Centre Hospitalier Rgional Universitaire de Besanon for their expertise with nephroblastoma and Loredane Vieille for achieving the manual segmentations. The authors thank European Community (European FEDER) for financing this work by the INTERREG V, the Communaut d'Agglomration du Grand Besanon and the Cancrople Grand-Est.

\section{References}

[1] Attig, A., Perner, P.: A study on the case image description for learning the model of the watershed segmentation. Transactions on Case-Based Reasoning 2(1), 41-53 (2009)

[2] Attig, A., Perner, P.: Incremental learning of the model for watershedbased image segmentation. In: Combinatorial Image Analaysis, pp. 209222. Springer (2012)

[3] Çiçek, Ö., Abdulkadir, A., Lienkamp, S.S., Brox, T., Ronneberger, O.: $3 \mathrm{~d}$ u-net: learning dense volumetric segmentation from sparse annotation. In: International Conference on Medical Image Computing and ComputerAssisted Intervention. pp. 424-432. Springer (2016)

[4] Cordier, A., Fuchs, B., Mille, A.: Engineering and learning of adaptation knowledge in case-based reasoning. In: EKAW. pp. 303-317. Springer (2006) 
[5] d'Aquin, M., Lieber, J., Napoli, A.: Adaptation knowledge acquisition: A case study for case-based decision support in oncology. Computational Intelligence 22(3-4), 161-176 (2006)

[6] Diaz, F., Fdez-Riverola, F., Corchado, J.M.: gene-cbr: A case-based reasonig tool for cancer diagnosis using microarray data sets. Computational Intelligence 22(3-4), 254-268 (2006)

[7] Dufour-Lussier, V., Le Ber, F., Lieber, J., Martin, L.: Adapting spatial and temporal cases. In: International Conference on Case-Based Reasoning. pp. 77-91. Springer (2012)

[8] Frucci, M., Perner, P., di Baja, G.S.: Case-based reasoning for image segmentation by watershed transformation. In: Case-Based Reasoning on Images and Signals, pp. 319-353. Springer (2008)

[9] Fuchs, B., Lieber, J., Mille, A., Napoli, A.: An algorithm for adaptation in case-based reasoning. In: Proceedings of the 14th European Conference on Artificial Intelligence. pp. 45-49. IOS Press (2000)

[10] Golobardes, E., Llora, X., Salamó, M., Martı, J.: Computer aided diagnosis with case-based reasoning and genetic algorithms. Knowledge-Based Systems 15(1), 45-52 (2002)

[11] Henriet, J., Chatonnay, P.: Introduction of a combination vector to optimise the interpolation of numerical phantoms. Expert Systems with Applications 40(2), 492-499 (2013)

[12] Henriet, J., Chatonnay, P., Leni, P.E.: An iterative precision vector to optimise the cbr adaptation of equivox. Engineering Applications of Artificial Intelligence 35, 158-163 (2014)

[13] Henriet, J., Lang, C.: Introduction of a multiagent paradigm to optimize a case-based reasoning system designed to personalize three-dimensional numerical representations of human organs. Biomedical Engineering: Applications, Basis and Communications 26(05), 1450060 (2014)

[14] Henriet, J., Leni, P.E., Laurent, R., Salomon, M.: Case-based reasoning adaptation of numerical representations of human organs by interpolation. Expert Systems with Applications 41(2), 260-266 (2014)

[15] Kato, Z., Zerubia, J., et al.: Markov random fields in image segmentation. Foundations and Trends@ in Signal Processing 5(1-2), 1-155 (2012)

[16] Kolodner, J.: Case-based reasoning. CA Morgan Kaufmann (1993)

[17] Lieber, J.: Application of the revision theory to adaptation in case-based reasoning: The conservative adaptation. Case-Based Reasoning Research and Development pp. 239-253 (2007) 
[18] Litjens, G., Kooi, T., Bejnordi, B.E., Setio, A.A.A., Ciompi, F., Ghafoorian, M., van der Laak, J.A., van Ginneken, B., Sánchez, C.I.: A survey on deep learning in medical image analysis. Med Image Anal 42, 66-88 (Dec 2017)

[19] Long, J., Shelhamer, E., Darrell, T.: Fully Convolutional Networks for Semantic Segmentation. arXiv:1411.4038v2 (2015)

[20] Marling, C., Montani, S., Bichindaritz, I., Funk, P.: Synergistic case-based reasoning in medical domains. Expert systems with applications 41(2), 249-259 (2014)

[21] Melis, E., Lieber, J., Napoli, A.: Reformulation in case-based reasoning. Advances in Case-Based Reasoning pp. 172-183 (1998)

[22] Montani, S.: Case-based reasoning for managing noncompliance with clinical guidelines. Computational Intelligence 25(3), 196-213 (2009)

[23] Perner, P.: An architecture for a cbr image segmentation system. Engineering Applications of Artificial Intelligence 12(6), 749-759 (1999)

[24] Perner, P., Attig, A.: Using prototype-based classification for automatic knowledge acquisition. In: Pattern Recognition, Machine Intelligence and Biometrics, pp. 197-212. Springer (2011)

[25] Thong, W., Kadoury, S., Piché, N., Pal, C.J.: Convolutional networks for kidney segmentation in contrast-enhanced ct scans. Computer Methods in Biomechanics and Biomedical Engineering: Imaging \& Visualization pp. 16 (2016)

[26] Trzupek, M., Ogiela, M.R., Tadeusiewicz, R.: Intelligent image content semantic description for cardiac 3d visualisations. Engineering Applications of Artificial Intelligence 24(8), 1410-1418 (2011)

[27] Wang, Z., Bovik, A.C., Sheikh, H.R., Simoncelli, E.P.: Image quality assessment: from error visibility to structural similarity. IEEE transactions on image processing $\mathbf{1 3}(4)$, 600-612 (2004) 